\title{
Comunicación / paz-conflicto: una relación en permanente tensión
}

Andrés Felipe Ortiz Gordillo

118 de octubre de 2012 se instaló en Oslo, Noruega, la mesa de ne-
gociación entre las Fuerzas Armadas Revolucionarias de Colombia (Farc-ep) y el Gobierno Nacional con sus respectivos representantes, que junto a los gremios y otros sectores de la sociedad exponían públicamente su visión de país y las orientaciones generales sobre los temas prioritarios para la finalización del conflicto armado y la consecución de la paz (un acuerdo resumido en cinco puntos: (1) Política de desarrollo agrario integral, (2) Participación política, (3) Fin del conflicto, (4) Solución al problema de las drogas ilícitas, (5) Víctimas y verdad). Como voceros hicieron sus intervenciones: Humberto de La Calle Lombana, por el Gobierno Nacional, y alias Iván Márquez, representante de las Farc-ep.

Al ser esta una noticia sustancial y prioritaria para el país, medios masivos de comunicación como RCN televisión ${ }^{1}$ y Caracol televisión ${ }^{2}$ decidieron, como política editorial, transmitir completamente

1 RCN Televisión hace parte del conjunto de empresas del grupo empresarial Ardila Lulle. Carlos Ardila Lulle se encuentra, según la revista Forbes, en el grupo de los 300 hombres más ricos del mundo.

2 Caracol Televisión es propiedad del grupo empresarial Santo Domingo. 
la intervención del vocero del gobierno, al tiempo que omitieron la transmisión del representante del grupo insurgente.

Esta circunstancia afectó de manera directa la posibilidad de los ciudadanos de acceder a una información amplia y plural, que referenciara los diferentes puntos de vista acerca del conflicto armado y social que se ha dado en el país en los últimos sesenta años, lo que lleva, en términos prácticos, a establecer una suerte de censura informativa, pues el discurso mediático se construye, de manera casi que exclusiva, desde uno y solo uno de los puntos de vista intervinientes en el proceso.

En principio esta política editorial promulgada de facto por algunos medios masivos hegemónicos de comunicación, afecta directamente los principios de equidad y pluralidad informativa, principios sobre los cuales se sustenta la dirección responsable de la opinión pública, y que consiste, como señala el Observatorio de Medios en Derechos Humanos, Medios al Derecho (MAD), en la responsabilidad que tienen los medios de

facilitar a los ciudadanos, por la vía de la información, argumentos que contribuyan a dar una mayor y mejor explicación de los contextos, actores, circunstancias, cumplimientos e incumplimientos de las partes implicadas, acuerdos históricos y sus resultados, entre otras cuestiones sobre los cuales se ha estructurado el conflicto colombiano. (2012a)

Al restringir los medios de comunicación masivos la posibilidad de que los ciudadanos accedan a la visión que del país tiene cada una de las partes en conflicto, se crea un escenario donde los referentes simbólicos sociales (que dependen en gran parte de la información que circula desde los medios de comunicación) propician validaciones críticas insuficientes de cada situación (en este caso del proceso de negociación de paz), lo que lleva a que se construya, por parte de la ciudadanía, una visión unilateral (oficialista, gobiernista) de los acontecimientos y de las decisiones públicas relacionadas con el conflicto y la paz.

Tal es la importancia de la información y la comunicación mass mediática, que uno de los importantes temas de debate en la mesa de negociación de La Habana, ha sido, en el punto de participación política, el de la "democratización de la información, de la comunicación 
y de los medios" que, al decir de las Farc-ep, debe comprender las siguientes iniciativas:

1. Participación social en el diseño, puesta en marcha y control de políticas de información y comunicación.

2. Democratización de la propiedad sobre los medios de comunicación y fortalecimiento de la propiedad pública estatal y comunitaria.

3. Democratización del espectro radioeléctrico y distribución equitativa de frecuencias de radio y televisión.

4. Derecho a la información veraz y oportuna, responsabilidad ulterior y derecho de réplica.

5. Acceso a los medios de comunicación de la oposición política y social.

6. Acceso especial a los medios de comunicación de comunidades campesinas, indígenas y afrodescendientes y de sectores sociales excluidos, en especial de las mujeres, los jóvenes y la comunidad LGTBI.

7. Financiación estatal y privada de medios de comunicación alternativos y comunitarios.

8. Acceso universal a las tecnologías de la información y las comunicaciones.

9. Mejoramiento de las condiciones laborales de los trabajadores de la información y la comunicación.

10. Programa especial de información y comunicación para la reconciliación y la construcción de la paz con justicia social. (Farc-ep, 2013)

Frente a este problemático panorama, en Colombia se han realizado estudios que dan cuenta de la relación medios de comunicación/ conflicto/esfera público-politica desde espacios institucionales, académicos y ciudadanos, algunos vinculados a universidades y otros desde organizaciones no gubernamentales, que tiene como resultado la producción de significativos trabajos como Lo que le vamos quitando a la guerra: medios ciudadanos en contextos de conflicto armado en Colombia, de la profesora Clemencia Rodríguez (2008), 
donde se establece una relación directa entre expresiones comunicativas mediáticas y emergencias ciudadanas en el marco del conflicto:

¡La guerra colombiana existe!, pero la sociedad ha sobrevivido más allá de sus políticos, guerreros y gobernantes porque ha ejercido la resistencia cultural en comunicación. Así, los medios ciudadanos han permitido que la gente cuente y se cuente desde su dignidad. $Y$ es que en Colombia, a diferencia de muchos otros contextos de guerra, lo cultural es lugar de encuentro y tiene una larga trayectoria de activismo mediático comunitario. (Rodríguez, 2008, p.3)

Numerosos estudios en Colombia han concentrado sus esfuerzos en determinar la responsabilidad que le corresponde a medios de comunicación y periodistas en el cubrimiento del conflicto armado que vive el país:

Algunos afirman que los comunicadores deben tomar partido y apoyar al gobierno como institución legítimamente constituida; (...) otros, por el contrario piensan que esta libertad debe protegerse y respetarse por encima de los intereses del gobierno, porque la responsabilidad de la prensa en este conflicto es mostrar la realidad. (Pachón, 2005)

Una buena parte de los estudios gestionados desde organismos internacionales como el PNUD han propuesto

aportar [elementos de análisis y gestionar espacios de debate público para] una mejor comprensión acerca del impacto que el conflicto armado ha tenido sobre los medios de comunicación y sobre el oficio periodístico, así como analizar los efectos que produce el trabajo informativo de los medios sobre el desarrollo del conflicto. De igual forma se busca escuchar por parte de los periodistas, lectores y expertos, sus propuestas y soluciones entablando un diálogo entre diferentes sectores de la sociedad. (PNUD, 2004) 
En términos generales, los estudios han estado concentrados en tres frentes: el primero tiene que ver con el abordaje analítico de los medios masivos de comunicación y del régimen comunicativo hegemónico en Colombia; el segundo se ha enfocado en estudios aplicados al lenguaje de la guerra (análisis de contenidos) y su inclusión en el discurso informativo (desde los contenidos que circulan por los medios masivos de comunicación); y el tercero sobre la construcción de las agendas mediáticas y sus impactos en la opinión pública.

En lo que refiere a experiencias alter comunicativas y de alter medios, y cómo ellos intervienen en las narraciones del conflicto, es poco lo que se ha dicho, aunque se encuentran experiencias significativas, como las resumidas en los documentos: Lo que le vamos quitando a la guerra. Medios ciudadanos en contextos de conflicto armado en Colombia (Rodríguez, et al, 2008), Ya no es posible el silencio. Textos, experiencias y procesos de comunicación ciudadana (Rincón, et al, 2007) y Pistas para narrar la paz. Periodismo en el posconflicto (Morelo, et al, 2014). Este enfoque de mirar la relación alter medios y conflicto ha sido desarrollado, fundamentalmente, por organizaciones no gubernamentales $(\mathrm{ONG})$, organizaciones comunitarias y populares, algunas de ellas relacionadas en sus actividades con temas inscritos en el marco del conflicto y la comunicación.

En esta exploración de los tránsitos conceptuales y analíticos que ha tenido el campo de la comunicación "en conflicto", encontramos experiencias que se dirigen a establecer cómo la relación de los medios de comunicación con el conflicto se da en contextos de tensión, y cómo los medios masivos se constituyen en agentes de desinformación:

En el cubrimiento de hechos relacionados con el conflicto interno que vive Colombia se observa que los periodistas con frecuencia descontextualizan las situaciones noticiosas, bien porque no investigan analizando causas y consecuencias, o porque se sienten amenazados por los actores armados y prefieren dar los menos datos posibles que favorezcan a que la información tenga antecedentes para claridad [de las audiencias]. (Correa, 2007, p.189) 
Son también amplios los estudios que se dirigen a establecer las relaciones de los medios de comunicación con la construcción de la esfera pública, pero pocos los que contemplan en su relación, dos elementos fundamentales en este estudio: los medios de comunicación en perspectiva comunitaria, alternativa, popular, ciudadana... (Alter medios), y el cubrimiento informativo que estos medios hacen del conflicto.

\section{Tránsitos y debates de la comunicación ¿para la paz?}

Teniendo en cuenta lo anterior, se puede señalar, a modo de síntesis analítica, que la participación de la comunicación y los medios en el conflicto armado (en el cubrimiento informativo) ha sido profundamente problemática. De cara a este escenario de tensiones, se pueden ubicar, en los últimos 15 años, cinco enfoques de discusión en los que se debate el papel de los medios de comunicación en el contexto del conflicto armado:

- El primer enfoque propone a los medios de comunicación como actores "neutrales y objetivos" en el tratamiento de informaciones relacionadas con el conflicto armado.

- El segundo inscribe a los medios de comunicación en la lógica del conflicto, y los señala como actores fundamentales del mismo.

- El tercer enfoque atiende a la necesidad de ver a los medios de comunicación como agentes de paz y promotores de los derechos humanos.

- El cuarto enfoque considera los impactos del discurso mediático en la opinión pública, y las tensiones que formula el escenario mass mediático a las libertades inherentes a la comunicación, la comunicación política y, en resumen, a la democracia.

- Un quinto enfoque se delimita en el interés de establecer los posibles "estándares de calidad" de la información y de su cumplimiento por parte de los actores de la comunicación mass mediática. 
Miremos ahora el desarrollo que ha tenido cada uno de estos enfoques, aclarando que la delimitación propuesta no excluye una mirada dialógica entre ellos. Lo que se valida en el proceso de análisis para establecer un estado de la cuestión sobre las reflexiones formuladas por diferentes actores en torno a la relación comunicación-conflicto-paz, son los énfasis propuestos en el proceso de interpretación. En este sentido, proponemos que la interpretación del presente estado de la cuestión tenga en cuenta que a pesar de lo esquemático del análisis (delimitado en 5 enfoques), existe la necesidad de profundizar en las relaciones que se establecen entre estos elementos, lo cual permitiré complejizar, sin duda, el debate aquí propuesto.

Una aclaración pertinente: los estudios relacionados con análisis de contenido / discursos son tomados desde su perspectiva metodológica. Esto quiere decir que los documentos relacionados con análisis de contenidos / discurso no se reseñan como un enfoque concreto en el mapa interpretativo propuesto, sino que se asumen, más bien, en función de los cinco enfoques establecidos, por cuanto su objetivo no es el análisis de contenido / discurso en sí mismos, sino la interpretación de campos de análisis vinculados con los enfoques, a partir del uso metodológico del análisis textual.

\section{Enfoque uno: la comunicación mass mediática y los medios son neutrales y objetivos}

El primer enfoque señala que los medios de comunicación no tienen ninguna responsabilidad en el conflicto, por cuanto su función les impone "ser objetivos" en el tratamiento de la información. Un acontecimiento hito que resume esta perspectiva, defendida de manera fundamental por los mismos periodistas, editores y directores de medios, es la declaración que se conoce como Acuerdo por la discreción $n^{3}$, suscrito en noviembre

3 El preámbulo del Acuerdo por la discreción señalaba lo siguiente: "Preferimos perder una noticia antes que una vida. El "Acuerdo por la discreción", un compromiso de todos. Por iniciativa de la Facultad de Comunicación Social y Periodismo de la Universidad de la Sabana (Bogotá), académicos y directores de medios de información de Colombia definieron el Acuerdo por la discreción, 
de 1999 por un grupo de 35 directores de medios de comunicación, quienes propusieron "elevar el nivel de calidad y responsabilidad en el cubrimiento y difusión de hechos violentos". Transcribimos a continuación el acuerdo, por considerarlo relevante para el análisis:

Bogotá, 4 de noviembre de 1999.

\section{Sobre la difusión de hechos violentos}

Conscientes de la responsabilidad social de nuestro oficio, los profesionales de los medios de comunicación de Colombia nos comprometemos con este Acuerdo por la discreción, porque queremos contribuir al logro de la paz, al respeto de la vida y a la búsqueda del bien común.

1. El cubrimiento informativo de actos violentos - ataques contra las poblaciones, masacres, secuestros y combates entre los bandos- será veraz, responsable y equilibrado. Para cumplir con este propósito, cada medio definirá normas de actuación profesional que fomenten el periodismo de calidad y beneficien a su público.

2. No presentaremos rumores como si fueran hechos. La exactitud, que implica ponerlos en contexto, debe primar sobre la rapidez.

3. Fijaremos criterios claros sobre las transmisiones en directo, con el fin de mejorar la calidad de esa información y evitar que el medio sea manipulado por los violentos.

4. Por razones éticas y de responsabilidad social no presionaremos periodísticamente a los familiares de las víctimas de hechos violentos.

inicialmente firmado por 35 directores de los medios más importantes del país. El objetivo: elevar el nivel de calidad y responsabilidad en el cubrimiento y difusión de hechos violentos. El Acuerdo propone que cada medio elabore un código ético, normas específicas o estándares profesionales que ayuden a mejorar la calidad de las informaciones sobre hechos violentos. La firma colectiva del Acuerdo por la discreción se llevó a cabo el 4 de noviembre de 1999 en Bogotá. Al acto fueron invitados todos los periodistas del país." (Reseñado en Serrano, 2006). 
5. Estableceremos criterios de difusión y publicación de imágenes y fotografías que puedan generar repulsión en el público, contagio con la violencia o indiferencia ante esta.

6. Respetaremos y fomentaremos el pluralismo ideológico, doctrinario y político. Utilizaremos expresiones que contribuyan a la convivencia entre los colombianos. Preferimos perder una noticia antes que una vida.

Esta declaración expresa, de manera transversal en la totalidad de los puntos y de manera particular en los tres primeros, que "la objetividad" es un criterio orientador del trabajo periodístico. Al respecto Serrano (2006) señala que:

El problema radica en que el texto no aclara qué quiere decir veraz, responsable, equilibrado (primer punto del acuerdo), exacto (segundo punto del acuerdo) y criterios claros (tercer punto del acuerdo), porque no especifica qué comportamientos profesionales y las situaciones en que dichos comportamientos conducirían a la veracidad, la responsabilidad y el equilibrio informativo. Con estos puntos, el Acuerdo por la discreción pone de manifiesto el contrato de comunicación informativo con el que los medios se comprometen a ofrecer una información “objetiva” (Serrano, 2006).

[...] En otras palabras, consideramos que el principal problema del Acuerdo por la discreción se halla en que propone un contrato informativo que genera falsas expectativas en la audiencia porque no está acorde con la práctica y porque no reconoce ante su público las limitaciones con las que debe trabajar: mientras exista confrontación armada, la presión de los actores armados no va a dejar de existir así los medios no lo deseen y traten de controlarlo, siempre serán víctimas en mayor o menor medida, voluntaria o involuntariamente, de la especialización en la concepción de estrategias comunicativas que los actores en conflicto implementarán en su lógica de guerra. Pretender desconocer este hecho es una falta de honestidad con ellos mismos y con los ciudadanos. Del mismo modo, el hecho de tomar partido por uno de los actores armados, por ejemplo por el Ejército, ya que representa la "legitimidad" del 
Estado, no implica que el medio de comunicación y sus periodistas no vayan a ser "víctimas" de las estrategias de comunicación que este actor armado utilizará para legitimar su posición deslegitimando la del enemigo. (Serrano, 2006)

Con todo, aquella mirada "objetivista" y "neutral” que esgrimen los medios de comunicación a sus audiencias, ha hecho carrera y se ha posicionado socialmente como legítima, a pesar de los múltiples interrogantes que genera. Tal y como señala Serrano (2006), este escenario de discusión hay que analizarlo a la luz de una serie de factores de orden económico, político, ideológico, tecnológico, de formación de audiencias críticas, etc., que son los que, sumados, definen y direccionan hoy el campo de la comunicación masiva y de los medios que la agencian socialmente.

En el marco de este enfoque se citan a continuación los documentos que se consideran más relevantes:

Tabla 1. Documentos del estado del arte, enfoque 1

\begin{tabular}{|c|c|}
\hline \multicolumn{2}{|c|}{ Enfoque 1} \\
\hline Referencia bibliográfica & Reseña \\
\hline $\begin{array}{l}\text { Roncancio, B. (2009). La } \\
\text { prensa en el conflicto armado. } \\
\text { Colombia 2003-2009. } \\
\text { Bogotá: Pontificia Universidad } \\
\text { Javeriana. }\end{array}$ & $\begin{array}{l}\text { Se destaca por los aportes de los } \\
\text { testimonios de nueve personajes } \\
\text { del ámbito público y con unas } \\
\text { características esenciales. Cada } \\
\text { uno desde su perspectiva, } \\
\text { conocimiento y postura realizó } \\
\text { una crítica al rol de la prensa } \\
\text { en el proceso de paz y Ley de } \\
\text { justicia y paz (Ley 975, 2005) } \\
\text { que construirán una mirada y } \\
\text { la realidad del periodismo en } \\
\text { Colombia. }\end{array}$ \\
\hline
\end{tabular}




\section{Enfoque dos: los medios de comunicación son un actor más del conflicto}

El segundo enfoque de análisis propone a los medios de comunicación como un actor más del conflicto armado. En el escenario de los conflictos bélicos contemporáneos, los medios de comunicación se constituyen en actores fundamentales: "son sin duda un actor de la guerra y a veces, pocas, un actor de la paz” (Barbero, 2000). Por su parte el Observatorio de Medios en Derechos Humanos, Medios al Derecho (MAD), señala que desde la sociedad civil se ha venido reflexionando sobre la relación que se establece entre los medios de comunicación, los derechos humanos, el conflicto armado y las culturas de paz, indicado que:

Por los medios de comunicación, que son dispositivos que administran el recurso simbólico de la información para el debate público, las sociedades tienen la posibilidad/imposibilidad de recoger elementos del saber colectivo referente a las cuestiones públicas, que permiten a los ciudadanos participar cualificadamente en el debate de las cosas que les son comunes. Por los medios se despliegan una serie de prácticas sociales y culturales que, dependiendo de la responsabilidad que se aplique a los procesos de producción informativa, pueden contribuir en el agenciamiento de una cultura de paz vinculada de manera profunda con el respeto de los derechos fundamentales, incluidos los derechos relativos a la comunicación, la información, la opinión y la expresión ciudadana o, por el contario, estarán dispuestos para la consolidación de la cultura de la guerra. (Medios al Derecho - MAD, 2012a)

Partimos de entender que desde los medios de comunicación se juega, de facto, un papel preponderante en la construcción de representaciones sociales sobre lo que acontece en el marco del conflicto y sus mediaciones, lo que implica situar la relación comunicación/medios -conflicto - construcción política de lo público en una red de interacciones, donde se establecen formas y sentidos de la realidad compartida, es decir, 
como señalan Berger y Luckmann, “en razón de lo que en las sociedades se da por establecido como conocimiento" (2008, p.15).

En el sentido de lo anterior, desde esta perspectiva de análisis se señala que es obligación de los medios de comunicación "construir un discurso mediático informativo plural, que implique a los medios la consulta, en equidad, de las diferentes voces participantes en el proceso [de negociación del conflicto], sean ellas voces institucionales, grupos insurgentes y sociedad civil." (MAD, 2012b), ya que el tratamiento restringido de la información que se configura al interior de los medios masivos de comunicación, afecta la posibilidad de la participación cualificada de los ciudadanos en el debate público sobre la paz en Colombia. Lo anterior porque, como señala Ignacio Ramonet,

la libertad de los medios de comunicación solo es la extensión de la libertad colectiva de expresión, fundamento de la democracia. Como tal, no puede ser confiscada por un grupo de poderosos. Implica, por añadidura, una 'responsabilidad social' y, en consecuencia, su ejercicio debe estar, en última instancia, bajo el control responsable de la sociedad... (2003)

En el marco de este enfoque se citan a continuación los documentos que se consideran más relevantes:

Tabla 2. Documentos del estado del arte, enfoque 2

\begin{tabular}{|l|l|}
\hline \multicolumn{1}{|c|}{ Referencia bibliográfica } & \multicolumn{1}{|c|}{ Enfoque 2 } \\
\hline $\begin{array}{l}\text { Domínguez, J. F. (2002). } \\
\text { Papel de los medios en el } \\
\text { proceso de consolidación } \\
\text { y expansión de las } \\
\text { FARC. Santiago de Cali: } \\
\text { Universidad del Valle. }\end{array}$ & $\begin{array}{l}\text { Los medios de comunicación en torno al conflicto } \\
\text { armado lejos de motivar la participación ciudadana } \\
\text { en función de la estructuración de la esfera de lo } \\
\text { público, ha contribuido a que la guerrilla de las } \\
\text { Farc-ep, puedan avanzar rápidamente en sus planes } \\
\text { de control territorial. Sin la ayuda de los medios } \\
\text { de comunicación hubiera sido imposible para esta } \\
\text { guerrilla, pensar seriamente en la posibilidad de ser } \\
\text { considerados como fuerza beligerante. }\end{array}$ \\
\hline
\end{tabular}




\begin{tabular}{|c|c|}
\hline $\begin{array}{l}\text { Chethuan, E. G. (2009). } \\
\text { El postconflicto en } \\
\text { Colombia: Una realidad } \\
\text { mediática. Bogotá, D.C: } \\
\text { Pontificia Universidad } \\
\text { Javeriana. }\end{array}$ & $\begin{array}{l}\text { Durante más de cuatro décadas de un conflicto } \\
\text { armado interno en Colombia, no se ha logrado un } \\
\text { consenso verdadero sobre lo vivido. Desde distintos } \\
\text { sectores se habla de la existencia de un conflicto } \\
\text { mientras que otros lo niegan rotundamente al } \\
\text { entender la violencia como un asunto de criminales } \\
\text { y bandidos. Por esta razón es que es problemático } \\
\text { afirmar ciertas hipótesis sin tener en cuenta que la } \\
\text { dinámica de la violencia está llena de altibajos y } \\
\text { que depende de factores externos e inconstantes. }\end{array}$ \\
\hline $\begin{array}{l}\text { Polo, R. A. (2013). } \\
\text { El manejo de los medios de } \\
\text { Comunicación al conflicto } \\
\text { armado. Colombia: } \\
\text { periodismodehoy.ning.com. }\end{array}$ & $\begin{array}{l}\text { Ilustra que los medios de comunicación han } \\
\text { sido utilizados desde su creación para informar } \\
\text { a la población, ya sea local, regional, nacional o } \\
\text { internacional, acerca del acontecer de los pueblos. } \\
\text { Así mismo, estos “instrumentos informáticos” por } \\
\text { divulgar las noticias de interés, han adquirido una } \\
\text { enorme preponderancia a lo largo de los años. } \\
\text { Como muestra de ello a la prensa se le denomina, } \\
\text { en numerosas ocasiones, como el Cuarto Poder. }\end{array}$ \\
\hline
\end{tabular}

\section{Enfoque tres: la comunicación y los medios son escenarios estratégicos para la paz}

Un tercer enfoque propone que los medios de comunicación deben actuar como "actor de la paz, en defensa de las víctimas y de los derechos humanos", más allá de los criterios que imponen los manuales sobre la neutralidad y la objetividad periodística. Esta es, en Colombia, una perspectiva de análisis y acción comunicativa relativamente nueva en la discusión pública nacional, a pesar de que su implementación se viene dando hace algunas décadas.

La orientación de "la comunicación en perspectiva de las víctimas", surge en el contexto de las reivindicaciones logradas por las organizaciones y el movimiento de víctimas del conflicto armado, movimiento campesino e indígena, ambientalistas, entre otros sectores sociales populares, y se posiciona como una opción renovadora del "discurso celebratorio" de la violencia y de los victimarios, así como del "discurso objetivista" que pretenden promover algunos actores vinculados a los medios de comunicación y algunos enfoques de la academia.

Se inaugura, desde este escenario, una plataforma comunicativa que procura señalar que los medios masivos de comunicación han sido 
"complacientes" con los actores del conflicto armado, en detrimento de las víctimas, quienes se redujeron, en el discurso mass mediático, a la condición de fuentes de tercera categoría (primero las fuentes oficiales, en segundo lugar los victimarios y, finalmente, las víctimas, que en realidad no eran fuente de información, sino objetos de espectacularización mediática).

Un ejemplo de esta "complacencia" lo encontramos en un fallo del Tribunal Superior de Bogotá, en la condena contra Salvatore Mancuso, dada en octubre de 2014, donde además de la sentencia al jefe paramilitar se presentan una serie de cuestionamientos al modo como los medios masivos de comunicación posibilitaron el posicionamiento del “discurso antisubversivo" en Colombia:

No menos sorprendente resultó el análisis del Tribunal sobre el papel de los medios de comunicación en el conflicto armado. Según él, los medios fueron "complacientes" con el paramilitarismo, toda vez que publicaron extensas entrevistas con el máximo jefe de las AUC, Carlos Castaño Gil, en las que el desaparecido fundador de las autodefensas habría publicitado su discurso antisubversivo. Una posición cuestionable, a la que añadió la opinión de que la prensa "cumple un rol fundamental en la propagación y legitimación de los discursos de odio" proferidos por algunos funcionarios públicos. (El Espectador, 31/10/2014. Subrayado fuera del texto original)

En este contexto, la plataforma comunicativa que se consolida desde la sociedad civil, activa una serie de críticas a los medios masivos de información, que son vistos como un actor más del conflicto armado. Las acciones que se despliegan desde ella promueven un trabajo en dos frentes: el primero tiene que ver con la gestión de medios propios $^{4}$; el

4 Como experiencias significativas podemos citar: el Colectivo de Comunicaciones Montes de María Línea 21 (Premio Nacional de Paz 2003), Agencia Prensa Rural (Premio Nacional de Paz 2010), el programa Contravía, el Tejido de comunicación de la ACIN, Producciones El Retorno de la Asociación Campesina de Antioquia, entre otras múltiples experiencias de alter medios. Para el caso de la ciudad de 
segundo frente tiene que ver con la generación de espacios de crítica a los medios masivos de comunicación, donde los alter medios y otras estrategias comunicativas como el performance, el teatro, el grafiti, la música, el audiovisual, entre otras expresiones artísticas y culturales, funcionaban como dispositivos sociales desde los cuales se promovían debates amplios sobre el régimen comunicativo hegemónico.

De este modo se consolida una plataforma comunicativa "para la paz", agenciada por múltiples medios locales y regionales, que busca hacer frente a lo que se ha denominado como un "cerco mediático" instituido por los medios masivos en relación con los temas de la guerra y de la paz.

Hay que decir también que este es un escenario de deliberación comunicativa que no se ha quedado en la mera denuncia del régimen comunicativo hegemónico, sino que se ha dirigido a la promoción e implementación de experiencias alter comunicativas renovadoras de las agendas público mediáticas de la dinámica paz / conflicto. Así las cosas, desde lo que aquí hemos llamado "plataforma comunicativa para la paz” se han promovido espacios de reflexión-acción que buscan motivar, entre otras, dos cuestiones fundamentales: primero, delimitar los posibles contextos que, en respuesta a las acciones informativas hegemónicas de los medios masivos de información, han posibilitado el surgimiento de experiencias informativas y comunicativas alternativas en zonas de conflicto armado. Segundo, dar cuenta de las alternativas que desde la alter comunicación se formulan para hacer frente a un contexto informativo local franqueado por la presencia de actores armados, circunstancia que hace reflexionar las lógicas de cubrimiento informativo y deliberación pública que se encuentran profundamente fragmentadas e intervenidas por la guerra.

Bogotá, la experiencia del Canal Capital, bajo la administración de Hollman Morris y Lisandro Duque, retomando los lineamientos del plan de desarrollo de la Bogotá Humana, es una muestra de este enfoque de medios implementados con el criterio de la defensa de las víctimas y la promoción de los DDHH. 
En el marco de este enfoque se citan a continuación, los documentos que se consideran más relevantes:

Tabla 3. Documentos del estado del arte, enfoque 3

\begin{tabular}{|c|c|}
\hline \multicolumn{2}{|r|}{ Enfoque 3} \\
\hline Referencia bibliográfica & Reseña \\
\hline $\begin{array}{l}\text { González, A., Rodríguez, } \\
\text { C. y otros (2008). Lo } \\
\text { que le vamos quitando a } \\
\text { la guerra. Bogotá, D.C: } \\
\text { University of Oklahoma. }\end{array}$ & $\begin{array}{l}\text { Adentrarse en el mundo de los medios ciudadanos } \\
\text { en Colombia es meterse en una complicada maraña } \\
\text { de vulnerabilidades y obstáculos. Incluso iniciativas } \\
\text { con un derrotero y un proyecto comunicativo } \\
\text { claro experimentan malos ratos que incluso a veces } \\
\text { parecería ponerlos en peligro de muerte. Es también } \\
\text { parte importante de esta historia. No para señalar } \\
\text { culpables, sino más bien para que, así como el país } \\
\text { debe apropiarse de estas iniciativas como suyas, } \\
\text { también nos responsabilicemos todos por asegurar } \\
\text { su futuro. }\end{array}$ \\
\hline $\begin{array}{l}\text { Rodríguez, C., Cadavid, } \\
\text { A. y Duran, O. (2008). } \\
\text { De la violencia al } \\
\text { discurso. Conflicto y } \\
\text { radios ciudadanas en } \\
\text { el Magdalena Medio. } \\
\text { Magdalena Medio: } \\
\text { Centro de competencia } \\
\text { en comunicación para } \\
\text { América Latina. }\end{array}$ & $\begin{array}{l}\text { En ese texto se documentó una red de quince } \\
\text { emisoras de radio ciudadana que actúa en la región } \\
\text { del Magdalena Medio (MM) ha sido exitosa } \\
\text { generando cambios en el tejido social y cultural, } \\
\text { y, trasladando el conflicto de la opción por la } \\
\text { violencia armada a la opción por la palabra (diálogo } \\
\text { y discurso). Estos medios ciudadanos han logrado } \\
\text { trasladar el conflicto del ámbito de la acción al } \\
\text { ámbito de las negociaciones y concertaciones } \\
\text { propias del espacio de la cultura. }\end{array}$ \\
\hline $\begin{array}{l}\text { Nieto. (2010). Relatos } \\
\text { autobiográficos de } \\
\text { víctimas del conflicto } \\
\text { armado: una propuesta } \\
\text { teórico-metodológica. } \\
\text { Bogotá, D.C: Universidad } \\
\text { de los Andes. }\end{array}$ & $\begin{array}{l}\text { El artículo avanza en la construcción de un marco } \\
\text { teórico-metodológico para valorarlo como estrategia } \\
\text { para la construcción de memorias. De esta manera, } \\
\text { el artículo une dos preguntas básicas: qué pasó } \\
\text { y cómo escribimos el relato de lo que pasó. La } \\
\text { primera funciona como una motivación para evocar, } \\
\text { recordar, enunciar y publicar relatos de los hechos } \\
\text { ominosos del pasado (estrategia de comunicación } \\
\text { y pedagogía); la segunda tiende a descubrir los } \\
\text { mecanismos de la narración propios de cada autor } \\
\text { (método autobiográfico y crítica genética). }\end{array}$ \\
\hline $\begin{array}{l}\text { Nieto. (2013). Medellín: } \\
\text { Relatos autobiográficos } \\
\text { del reciente de la ciudad de } \\
\text { Medellín conflicto armado } \\
\text { en Colombia el caso. } \\
\text { Medellín: Universidad } \\
\text { Nacional de La Plata. }\end{array}$ & $\begin{array}{l}\text { Un trabajo de reflexión sobre cómo narrar la } \\
\text { guerra desde una perspectiva diferente a la del } \\
\text { periodismo de titulares; tiene como base un modelo } \\
\text { metodológico en talleres de escritura dirigidos por } \\
\text { periodistas con el propósito de encontrar caminos } \\
\text { para contar la guerra desde adentro. }\end{array}$ \\
\hline
\end{tabular}




\section{Enfoque cuatro: comunicación para la democracia y fortalecimiento de lo público}

En el cuarto enfoque se consideran los impactos del discurso mediático en la opinión pública y en el ejercicio de la democracia participativa. Los estudios relacionados atienden a varias hipótesis, entre las que se cuentan aquellas que señalan que los medios de comunicación "siguen empeñados en la idea de que representan a la opinión pública y no, como sugiere Ana María Millares, que son un medio para que esa opinión se exprese" (Dominguez, 2002).

En este enfoque se señala el campo de la comunicación, de los medios y de la información como estratégico para el redimensionamiento de la política, y el agenciamiento de la esfera pública desde la participación comunicativa, lo que implica un análisis profundo de las realidades nacionales vinculadas al conflicto: sus actores y contextos, sus impactos, las posibles soluciones que se agencian desde la ciudadanía y no solo desde los escenarios hegemónicos de poder.

Esto implica asumir en perspectiva política la definición de las agendas mediáticas y de los enfoques instituidos para el cubrimiento de información pública, así como reconocer las dificultades, de múltiple tipo, que enfrentan los productores de la información: acceso a fuentes, situación laboral, formación, dificultades para hacer investigación, etc.

Por otro lado se señala la necesidad de superar la dinámica “del consenso informativo", por considerarse nocivo para una sociedad en crisis que busca alternativas por fuera de las dinámicas socio políticas, económicas y de conflicto actuales. Esto debe configurar un nuevo régimen de visibilidades públicas, donde las víctimas, los defensores de derechos humanos, las organizaciones y movimientos sociales, entre otros actores sociales, tengan autonomía en el acceso y uso de los medios que les posibiliten participar de la agenda público-comunicativa nacional. 
En el marco de este enfoque se citan a continuación los documentos que se consideran más relevantes:

Tabla 4. Documentos del estado del arte, enfoque 4

\begin{tabular}{|c|c|}
\hline \multicolumn{2}{|r|}{ Enfoque 4} \\
\hline $\begin{array}{c}\text { Referencia } \\
\text { bibliográfica }\end{array}$ & Reseña \\
\hline $\begin{array}{l}\text { Uribe. (2009). } \\
\text { Bogotá, D.C: } \\
\text { Medios y estrategias } \\
\text { de comunicación en } \\
\text { el conflicto armado } \\
\text { Colombiano. } \\
\text { Bogotá: Universidad } \\
\text { de los Andes. }\end{array}$ & $\begin{array}{l}\text { Para hablar del papel de los medios de comunicación } \\
\text { en el conflicto armado colombiano hay que analizar } \\
\text { tres aspectos: en primera medida es necesario entender } \\
\text { las estrategias de comunicación política del gobierno en } \\
\text { turno y la forma en que los medios hacen parte de dicha } \\
\text { dinámica; en segundo lugar hay que analizar el rol que } \\
\text { juegan los medios a la hora de informar sobre la guerra, es } \\
\text { decir, la presión e influencia sobre la sociedad civil y sobre } \\
\text { el gobierno; por último se deben examinar las garantías } \\
\text { para informar de una manera independiente el conflicto. }\end{array}$ \\
\hline $\begin{array}{l}\text { Tamayo y Bonilla. } \\
\text { (2013). El deber } \\
\text { de la memoria. La } \\
\text { agenda investigativa } \\
\text { sobre la cobertura } \\
\text { informativa del } \\
\text { conflicto armado en } \\
\text { Colombia, 2002- } \\
\text { 2012. Bogotá: } \\
\text { Universidad EAFIT. }\end{array}$ & $\begin{array}{l}\text { Esta obra ofrece una mirada interpretativa de las } \\
\text { principales tendencias que han guiado el estudio sobre la } \\
\text { relación entre los medios de comunicación, el periodismo } \\
\text { y la confrontación armada en Colombia entre } 2002 \text { y } \\
2012 \text {. ¿Cuál es la agenda investigativa que la academia, } \\
\text { las organizaciones sociales y de periodistas han elaborado } \\
\text { sobre esta problemática? El estudio reúne } 89 \text { trabajos } \\
\text { que se pueden agrupar en tres tendencias principales: } \\
\text { las miradas al oficio: riesgos, libertades y garantías para } \\
\text { informar en medio del conflicto; las agendas del conflicto: } \\
\text { fuentes, lenguajes y públicos de la información, y los } \\
\text { escenarios emergentes: (pos) conflicto, víctimas y memoria. }\end{array}$ \\
\hline $\begin{array}{l}\text { Bonilla, J.I. (2002). } \\
\text { Periodismo, } \\
\text { guerra y paz, } \\
\text { campo intelectual } \\
\text { periodístico y } \\
\text { agendas de la } \\
\text { información en } \\
\text { Colombia. México: } \\
\text { Universidad } \\
\text { Autonoma. }\end{array}$ & $\begin{array}{l}\text { Propone un análisis de las agendas informativas sobre la } \\
\text { guerra y la paz en Colombia, a partir de un doble ejercicio } \\
\text { teórico. Por una parte, se basa en la comprensión de la } \\
\text { esfera pública como una arena de "competencia política" } \\
\text { donde confluyen y se yuxtaponen diversos intereses, } \\
\text { marcos de acción e interpretación para acceder y significar } \\
\text { la realidad. Y por la otra, en el análisis del "campo" } \\
\text { periodístico como un espacio en tensión donde se llevan } \\
\text { a cabo una serie de interacciones y disputas entre las } \\
\text { instituciones mediáticas (y agentes profesionales llamados } \\
\text { periodistas) y otras esferas del poder de la sociedad } \\
\text { (políticos, ciudadanos, guerreros, organizaciones sociales, } \\
\text { empresarios, entre otros) por definir y redefinir los temas } \\
\text { y problemas que son objeto de atención pública sobre la } \\
\text { guerra y la paz en este país. }\end{array}$ \\
\hline
\end{tabular}




\begin{tabular}{|c|c|}
\hline $\begin{array}{l}\text { Bonilla y Montoya. } \\
\text { (2003). Periodistas, } \\
\text { politicos y } \\
\text { guerreros. Tres } \\
\text { hipótesis sobre } \\
\text { la visibilidad } \\
\text { mediática de la } \\
\text { guerra en Colombia. } \\
\text { Colombia: } \\
\text { Universidad de los } \\
\text { Andes. }\end{array}$ & $\begin{array}{l}\text { Establece una discusión teórica con algunos de los } \\
\text { conceptos más hegemónicos que han establecido los } \\
\text { alcances y los límites del campo intelectual y profesional } \\
\text { del periodismo en este tipo de situaciones. }\end{array}$ \\
\hline $\begin{array}{l}\text { Bonilla y Tamayo. } \\
\text { (2007). La violencia } \\
\text { en los medios y } \\
\text { los medios en las } \\
\text { violencias. Bogotá: } \\
\text { Universidad } \\
\text { Javeriana, Eafit, } \\
\text { Cinep, Colciencias. }\end{array}$ & $\begin{array}{l}\text { Realiza un estado del arte de documentos relacionados con } \\
\text { la relación violencia - medios de comunicación en América } \\
\text { Latina. Llama la atención en torno a la responsabilidad } \\
\text { pública de la investigación en sociedades caracterizadas } \\
\text { por una doble condición: la universalidad de las normas } \\
\text { y la fragmentación de los vínculos sociales. Retoma } \\
\text { postulados de intelectuales como DaMata, quien habla a } \\
\text { favor de una "antropología de la violencia» que sea capaz } \\
\text { de investigarla como un fenómeno inherente a la condición } \\
\text { humana para, luego, discutir sus manifestaciones en } \\
\text { sistemas sociales específicos, y cuya postura sería } \\
\text { comprender la violencia en sus aspectos universales y sus } \\
\text { dinámicas locales. }\end{array}$ \\
\hline $\begin{array}{l}\text { Montoya, L.C. } \\
\text { (2009). Medios de } \\
\text { comunicación y } \\
\text { organizaciones de } \\
\text { la sociedad civil. } \\
\text { Fortaleciendo } \\
\text { alianzas para la } \\
\text { democracia y los } \\
\text { derechos humanos } \\
\text { en América } \\
\text { Latina. Colombia: } \\
\text { Pontificia } \\
\text { Universidad } \\
\text { Javeriana. }\end{array}$ & $\begin{array}{l}\text { El presente artículo recoge los resultados de una } \\
\text { investigación financiada por la embajada del Reino } \\
\text { Unido en Colombia que recopila, por primera vez, } \\
\text { algunas experiencias en América Latina y Colombia de } \\
\text { alianzas entre medios y OSC, en pro de la democracia } \\
\text { y los derechos humanos. A través de entrevistas con } \\
\text { algunos de sus protagonistas, el estudio identifica retos } \\
\text { y lecciones para futuras alianzas. Así ayuda a fortalecer } \\
\text { la contribución de los medios de comunicación a la } \\
\text { democracia y los derechos humanos en Colombia. }\end{array}$ \\
\hline
\end{tabular}




\begin{tabular}{|l|l|}
\hline & $\begin{array}{l}\text { Destaca la forma en que la ofrenda de vidas, libertades y } \\
\text { bienes que los periodistas colombianos hacen cada año } \\
\text { en cumplimiento de su deber se repite dramáticamente. } \\
\text { Casi milimétricamente, como una cuota fija: 108 }\end{array}$ \\
Morales, M. (2013). & $\begin{array}{l}\text { casos de violaciones a la libertad de expresión y 167 } \\
\text { víctimas contabiliza la Fundación para la Libertad } \\
\text { de la Prensa (FLIP) de 2013 a la fecha. Hay una } \\
\text { de expresión: a } \\
\text { romper la cadena. } \\
\text { tendencia que rebasó las fronteras tercermundistas y las } \\
\text { analógicas para amenazar a las sociedades industriales } \\
\text { Razonpublica.com. } \\
\text { y al ciberperiodismo. Aquí, el aire está enrarecido y el } \\
\text { vecindario está convulsionado: Argentina y Ecuador, } \\
\text { con nuevas legislaciones; Venezuela y Cuba, con asedios } \\
\text { gubernamentales a los comunicadores, y hasta Estados }\end{array}$ \\
Unidos con su restricción en el acceso a la información \\
pública.
\end{tabular}

\section{Enfoque cinco: estándares de calidad informativa y situación actual del periodismo, los medios y la comunicación}

Este enfoque es el que tiene un mayor desarrollo, por cuanto se concentra en el análisis de "la práctica comunicativa y mediática”. Los estudios rastreados y analizados permiten establecer la existencia de una preocupación general por parte de la academia, las organizaciones y movimientos sociales, y productores de medios sobre la necesidad de concretar en la práctica unos estándares mínimos en los procesos de cubrimiento informativo relacionado con el conflicto armado y la paz.

Se señala, de manera general en los estudios, que existen efectivamente criterios de calidad expresados en manuales de redacción y acuerdos entre los actores del gremio informativo, pero estos acuerdos por lo general no se aplican en la cotidianidad de los medios de información. 
En el marco de este enfoque se citan a continuación los documentos que se consideran más relevantes:

Tabla 5. Documentos del estado del arte, enfoque 5

\begin{tabular}{|c|c|}
\hline \multicolumn{2}{|c|}{ Enfoque 5} \\
\hline Referencia bibliográfica & Reseña \\
\hline $\begin{array}{l}\text { Olano, R.A. (2008). Reflexiones } \\
\text { sobre el cubrimiento del diario El } \\
\text { Tiempo al conflicto armado: un } \\
\text { análisis desde la perspectiva de } \\
\text { la educación para la paz. Bogotá: } \\
\text { Pontificia Universidad Javeriana. }\end{array}$ & $\begin{array}{l}\text { Hace un análisis desde la perspectiva de } \\
\text { la "educación para la paz", en la cual } \\
\text { se reconocen unas funciones específicas } \\
\text { que deben de cumplir los medios de } \\
\text { comunicación y los periodistas en } \\
\text { particular, como trabajadores de la paz. } \\
\text { Hay que anotar que estas funciones } \\
\text { pueden considerarse de mediación, mas no } \\
\text { pueden limitarse dentro de esta palabra. }\end{array}$ \\
\hline $\begin{array}{l}\text { Bonilla, Tamayo y Gómez. (2005). } \\
\text { La televisión del conflicto. Bogotá: } \\
\text { Proyecto Antonio Nariño. }\end{array}$ & $\begin{array}{l}\text { Indaga sobre la presencia o la ausencia de } \\
\text { algunos estándares de calidad informativa } \\
\text { en el cubrimiento del conflicto armado, } \\
\text { mediante las dos siguientes preguntas de } \\
\text { investigación: ¿qué criterios de calidad } \\
\text { periodística asociados a la imparcialidad, } \\
\text { la pluralidad, la precisión, la claridad y } \\
\text { la diversidad de fuentes, temas y puntos } \\
\text { de vista están presentes o ausentes en las } \\
\text { agendas de los noticieros de televisión que } \\
\text { dan cuenta del conflicto armado interno?, } \\
\text { ¿qué prácticas profesionales y condiciones } \\
\text { sociales de producción noticiosa inciden } \\
\text { en la calidad de la información sobre esta } \\
\text { problemática? }\end{array}$ \\
\hline $\begin{array}{l}\text { Camargo y Blanco. (2007). Voces } \\
\text { y silencios sobre el desplazamiento } \\
\text { forzado en santa marta. Análisis } \\
\text { de la información ofrecida por los } \\
\text { medios de comunicación locales del } \\
\text { año } 2001 \text { al 2005. Santa Marta: } \\
\text { Universidad Sergio Arboleda, } \\
\text { Escuela de Comunicación Social y } \\
\text { Periodismo. }\end{array}$ & $\begin{array}{l}\text { La investigación ha sido complementada } \\
\text { con entrevistas a los periodistas } \\
\text { y directivos de diferentes medios } \\
\text { locales, para esclarecer aún más esta } \\
\text { problemática, e identificando los vacíos } \\
\text { en la comprensión de los fenómenos } \\
\text { del desplazamiento forzado, una de las } \\
\text { dificultades sociales más arraigadas } \\
\text { de nuestra región, con barrios enteros } \\
\text { de personas desplazadas que viven } \\
\text { en situaciones infrahumanas, y una } \\
\text { sociedad que no tiene claridad sobre la } \\
\text { verdadera dimensión e implicación de la } \\
\text { problemática actual. }\end{array}$ \\
\hline
\end{tabular}


Alarcón, R.J. (2001). Descripción y cuantificación del contenido informativo sobre conflicto armado colombiano en Radionet durante el primer semestre del 2001. Bogotá: Corporación Universitaria Minuto de Dios.

El Tiempo. (2003). El conflicto armado en las páginas de El Tiempo. Bogotá: El Tiempo.

Bonilla. (2003). Calidad informativa y cubrimiento del conflicto. Bogotá: Fundación Nuevo Periodismo Iberoamericano.
El estudio se inscribe en una línea de investigación de periodismo y medios, que además de enriquecer la bibliografía existente sobre radio, también ayudará a los profesionales de la comunicación a establecer qué tipo de información radial se podría llegar a manejar en el futuro, con base en la experiencia brindada por Radionet. Con la descripción y cuantificación del contenido informativo sobre el conflicto armado colombiano en este medio de comunicación durante el primer semestre de 2001 se deja abierta la puerta a la realización de una investigación en al cual se desarrolle el análisis de contenido desde las siguientes perspectivas: tratamiento de la información, ética, explicación de los hechos, seguimiento de la información.

El tema de responsabilidad social de los medios es observado como la forma en que se presentan en sus páginas temas tan cruciales como el conflicto interno que vive el país. Este primer cuaderno de análisis muestra, paso a paso, el recorrido adelantado en el periódico para describir y analizar su representación de la guerra y de la paz, en un periodo de tiempo complejo y difícil.

El estudio indagó sobre la presencia y los modos de articulación de algunos estándares de calidad informativa en la cobertura del conflicto armado en Colombia. La pregunta que este trabajo trato de responder fue la siguiente: ¿qué criterios de calidad periodística asociados a la pluralidad, la precisión, el manejo diverso de fuentes y temas de la información son los que aparecen en las páginas de los diarios en relación al conflicto armado interno? 
Castillo y Peñaranda. (2010). Tratamiento periodístico dado a las víctimas civiles del conflicto interno armado por el periódico El Tiempo, caso Íngrid Betancourt. Bogotá: Universidad Sergio Arboleda.
Se basa en el análisis de notas periodísticas mediante actos de habla, con el fin de determinar la intención del hablante y caracterizar el tratamiento mediático dado a víctimas específicas del conflicto armado colombiano, en este caso a la ex candidata presidencial Íngrid Betancourt.

$\mathrm{El}$ artículo tiene como referencia las declaraciones del desmovilizado Joice Hernández, en donde indica la ubicación de la fosa con el cuerpo del director de regalías de Córdoba, Jairo Alberto Zapa Pérez, quien era la mano derecha del gobernador de córdoba Alejandro Lyons. Los periodistas que se enfrentan a los gobiernos locales conocen bien los trucos que estos utilizan para censurar, y que no solo se limitan a la extorsión de la pauta publicitaria. Ocurre en Montería, Buenaventura, Tumaco, Caucasia, Barrancabermeja, Cúcuta, y así hasta nombrar la mayoría de las ciudades colombianas.

Medios colombianos de diferente peso y altura debaten sobre el papel que juegan en el camino hacia la paz transformadora:

Marcos, J. (2013). El periodismo colombiano a la luz del conflicto armado. Colombia: despazados.org/ cuadernos de periodismo. nuevos y tradicionales, masivos y alternativos, confesos partidistas y objetivos. El propio presidente Juan Manuel Santos ejerció la profesión, sobre la que su antecesor en el cargo, Álvaro Uribe, afirmó que solo hay dos tipos de periodistas: los enemigos y los amigos del terrorismo.

\section{En síntesis...}

Esta investigación se suscribe, entonces, en las lógicas y dinámicas propias del tercer enfoque analítico, en el que se propone ver a los medios de comunicación (a los alter medios y los procesos de alter comunicación) como agentes de paz y promotores de los derechos humanos. Este enfoque se presenta como eje fundamental del trabajo 
alter comunicativo implementado por las experiencias participantes, al tiempo que se complementa con elementos propios de otros enfoques que, como se ha señalado, inciden en la configuración de los procesos alter comunicativos.

Así, el enfoque suscrito se articula con una reflexión sobre los impactos del discurso mediático en la opinión pública, y sobre las tensiones que la comunicación formula a la democracia (enfoque cuatro). Al tiempo, el enfoque comunicativo que reivindica a los alter medios como actores de la paz evidencia preocupaciones relacionadas con los estándares de calidad de la información, por cuanto se evidencia una preocupación fundamental por cumplir con los rigores periodísticos en el tratamiento de la información (enfoque cinco). 